\title{
Influence of Weather Factors on Seasonal Population Dynamics of Coelaenomenodera elaeidis (Coleoptera: Chrysomelidae) and Its Natural Enemies in NIFOR, Nigeria
}

\author{
T. I. Aneni ${ }^{1 *}$, C. I. Aisagbonhi ${ }^{1}$, B. N. Iloba ${ }^{2}$, V. C. Adaigbe ${ }^{1}$, C. O. Ogbebor ${ }^{1}$ \\ ${ }^{1}$ Entomology Division, Nigerian Institute for Oil Palm Research, Benin City, Nigeria; ${ }^{2}$ Department of Animal and Environmental \\ Biology, University of Benin, Benin City, Nigeria. \\ Email: tomaneni1@yahoo.com
}

Received September $26^{\text {th }}, 2013$; revised November $18^{\text {th }}, 2013$; accepted December $3^{\text {rd }}, 2013$

Copyright (C) 2014 T. I. Aneni et al. This is an open access article distributed under the Creative Commons Attribution License, which permits unrestricted use, distribution, and reproduction in any medium, provided the original work is properly cited. In accordance of the Creative Commons Attribution License all Copyrights (C) 2014 are reserved for SCIRP and the owner of the intellectual property T. I. Aneni et al. All Copyright (C) 2014 are guarded by law and by SCIRP as a guardian.

\section{ABSTRACT}

The leaf miner (Coelaenomenodera elaeidis) is the major pest of the oil palm. Seasonality of C. elaeidis, its natural enemies and their relationship with temperature, rainfall and relative humidity were observed between January 2009 and December 2010 at the main station of the Nigerian Institute for Oil Palm Research. Leaf miner population estimates were obtained from NIFOR entomology division from 1976-1980. This study analyses temporal patterns in leaf miner abundance, and elucidates general patterns and factors influencing leaf miner abundance. Multiple linear regressions were used to analyse the relationship between abundance of leaf miner, its parasitoids and predators and the following climatic variables: maximum and minimum temperature $\left({ }^{\circ} \mathrm{C}\right)$, rainfall ( $\mathrm{mm}$ ) and relative humidity (\%). Climate variables from the month of pest collection (control variable) or from the month before collection (delayed variable) were used. The abundance of leaf miner and predatory ants peaked in the dry season, while parasitoids were most abundant in the rainy season. Significant correlations $(P \leq$ 0.05) were found between leaf miner, its natural enemies and both control and delayed weather variables. For all years, maximum temperature was the most dominant for all the leaf miner stages. This indicates that the weather variables at both the month of collection and with a delayed month in relation to collection are critical for pest-weather evaluation and important for leaf miner control. Temperature, rainfall and relative humidity had an effect on the population of $C$. elaeidis, and this effect is manifested primarily in seasonal fluctuations in oil palm agroecosystems. Weather influenced the seasonal population dynamics of $C$. elaeidis, facilitating early season build-up on the oil palm host crop. Dry season months with resultant higher temperatures recorded higher population of $C$. elaeidis.

\section{KEYWORDS}

Seasonality; Coelaenomenodera elaeidis; Oil Palm, Weather Variables; Pest Management

\section{Introduction}

Seasonal variation in abundance of tropical insects is a common phenomenon [1,2]. Insect abundance can change over time for a variety of reasons, including macroclimatic and microclimatic changes, and variation in the availability of food resources [1]. The effect of climate

\footnotetext{
${ }^{*}$ Corresponding author.
}

change in Nigeria is already contributing to extreme weather events: amount of rainfall, proliferation of pests, crop diseases and high temperature effects [3]. Insects are able to function faster and more efficiently at higher temperatures. They can feed, develop, reproduce, and disperse when the climate is warm, though they may live for a shorter time [4]. From an ecological point of view, particular variables such as the annual minimum temper- 
ature [5-7] or temperature in specific months [8-10] may be more important than the annual mean. Many biological processes undergo sudden shifts at particular threshold values [11-14]. Existing studies suggest that direct effects of temperature are likely to be larger and more important than any other factor [15]. In oil palms, the central role of natural balance in the control of leafeating insects continues to be recognized and investigated, with emphasis on selective control measures applied only in response to census counts. In a review of integrated pest management (IPM) developments, Ho and Teh [16] describe improving knowledge of technical aspects of the response system, and the relationship of known natural enemies to their flower food plants. Information on weather influences on leaf miner; its parasitoid and predator abundance patterns have not been obtained.

This study analyses temporal patterns in leaf miner abundance and its natural enemies, and elucidates general patterns and factors influencing leaf miner abundance.

\section{Materials and Methods}

\subsection{Study Site}

The study site is located at the main station of the Nigerian Institute for Oil Palm Research (NIFOR). It lies on the coordinates of latitude $6^{\circ} 30^{\prime} \mathrm{N}$ and longitude $5^{\circ} 40^{\prime} \mathrm{E}$. It is located in the forest zone of South-West Nigeria. The natural vegetation has been replaced by oil palm and coconut cultivation during the past 50 years. The study site was planted in 2000, whose crown canopy had not formed a continuous layer and sunlight could still penetrate to the ground.

\subsection{Climate}

There are two seasons: wet and dry seasons. Average mean temperature is $26.6^{\circ} \mathrm{C}$.

\subsection{Soils}

The study site and greater part of the Nigerian palm belt, both wild and planted, is on the "Acid Sands" soils [17]. These are developed on tertiary and cretaceous sediments, and the most recent parts, on which most of the palms grow, are largely unconsolidated sandstones or "Benin sands". These soils were classified as "fascs" [18] which are accepted as equivalent to soil families in present terminology. Under the soil taxonomy system, they are $\mathrm{Pa}-$ leudults and dystropepts, and under the FAO-UNESCO [19] system, they are dystric nitisols and dystric cambisols [20].

\subsection{Sampling Technique}

The study involved simple random sampling surveys for leaf miner on a plot 9 years old. Data was collected monthly from January 2009-December 2010. It involved observing and counting of leaf miner and its natural enemies. No pesticides were applied during the study period, purposely to simulate a natural ambience in the sample plot. A sampling intensity of 21 palms was used, selecting 1 palm per line. The larval, pupal and adult stages of the $C$. elaeidis were counted. The independent variables were temperature, rainfall and relative humidity. The dependent variable was Coelaenomenodera elaeidis counts and its natural enemies. At each point, C. elaeidis were counted on fronds inclining at $45^{\circ}$ (number 17 and 25 on the phyllotactic spiral). In shorter palms, fronds were pulled down by a stick, but in taller ones a ladder was used. A different palm was used at successive counts.

The plot was planted in 2000 and comprised of 443 palms (2.95 hectares). Harvesting of plot 54 began in 2005. Leaf miner counts on the palm leaflets within the field plot. NIFOR palms are planted in a triangular pattern, so census lines ran in three directions. Access points were marked with reference to field boundaries and harvesting paths. The most common predatory ants, Micromischoides sp., were identified and counted. Census on the basis of damage was done monthly by walking the full length of a planted line, assessing damage on each palm and cutting 5 severely damaged leaflets from a palm frond with a harvesting knife. The leaflets were opened up in the laboratory and immature stages of $C$. elaiedis were counted.

Parasitoids of C. elaeidis were identified and counted using the direct count method. Sampling was conducted monthly between 7 - $11 \mathrm{am}$. Methods for insect sampling included use of insect sweep net, direct handpicking and leaflet sampling.

\subsection{Past Data Collection}

Leaf miner past population estimates were obtained from NIFOR entomology division from 1976-1980.

\subsection{Statistical Analysis}

Multiple linear regressions were used to analyse the relationship between abundance of leaf miner, its parasitoids and predators and the following climatic variables: maximum and minimum temperature $\left({ }^{\circ} \mathrm{C}\right)$, rainfall $(\mathrm{mm})$ and relative humidity (\%). For the analyses, climate variables from the month of collection (control variable) or from the month before the collection (delayed variable) were used.

\section{Results}

Table 1 shows relationship between mean weather fac- 
tors and leaf miner insect stages, predators and parasitoids for 2009-2010. In 2009, adult leaf miner had a significant relationship for both control (0.008) with maximum temperature and relative humidity being the dominant variables and delayed (0.007) with rainfall being the dominant variable. Parasitoids had a significant relationship for control (0.001) with maximum temperature, rainfall and relative humidity all dominant variables. There was a significant relationship for grouped leaf miner stages in the control (0.039) and delayed (0.029) in 2009 with rainfall being the dominant variable.

In 2010, adult leaf miner had a significant relationship for control (0.048) with relative humidity being the dominant variable. Parasitoids had a significant relationship for delayed (0.047) with minimum temperature being the dominant variable. Predators had a significant relationship for delayed (0.001) with minimum temperature being the dominant variable. Table 2 shows rainy season relationship between mean weather factors and leaf miner insect stages, predators and parasitoids for 2009-2010. In 2009, Predators had a significant relationship for control (0.029) with relative humidity being the dominant variable. In 2010, there was no recorded dominant variable during this period. Table 3 shows dry season relationship between mean weather factors and leaf miner insect stages (larvae, pupae and adult), predators and parasitoids for 2009-2010. For both years, maximum temperature was the most dominant variable for all the leaf miner stages, predators and parasitoids. Table 4 shows relationship between mean weather factors and leaf miner insect stages for 1976-1980. In 1976, larvae had a significant relationship for delayed (0.041) with maximum temperature being the dominant variable. In 1980, larvae had a significant relationship for control (0.044) with minimum temperature being the dominant variable. In 1976, pupae had a significant relationship for control (0.028) with maximum and minimum temperature being the dominant variables and delayed (0.019) with maximum temperature being the dominant variable. In 1980, pupae had a significant relationship for delayed (0.015) with rainfall, maximum and minimum temperature being the dominant variables. Table 5 shows rainy season relationship between mean weather factors and leaf miner insect stages for 1976-1980. In 1979, larvae had a significant relationship for control (0.046) with relative humidity, maximum and minimum temperature being the dominant variables. Also in 1979, pupae had a significant relationship for control (0.024) with rainfall, relative humidity and maximum temperature being dominant variables during this period. In 1980, pupae had a significant relationship for control (0.042) with maximum temperature being dominant variable. Table 6 shows dry season relationship between mean weather factors and leaf miner insect stages for 1976-1980. For all years, maximum temperature was the most dominant variable for all the leaf miner stages.

\section{Discussion}

An understanding of the interactions between insect pests, temperature, rainfall and relative humidity would help in developing improved pest control strategies. There was a higher relationship between leaf miner and weather factors during the 2009-2010 periods (Table 1) in comparison with the 1976-1980 periods (Table 5). This could be attributed to higher mean temperatures prevailing at the 2009-2010 periods. This also implies that progressively higher temperatures, lower rainfall and relative humidity values could be implicated in the increase in leaf miner abundance. If these patterns continue, prediction of leaf miner response to climate variability will have to account for the magnitude and timing. Weather controls the development rate, survival, fitness, and level of activity of individual insects: the phenology, distribution, size, and continuity of insect populations; migration and their es-

Table 1. Relationship between mean weather factors and leaf miner stages (larvae, pupae, adult), predators and parasitoids for 2009-2010.

\begin{tabular}{|c|c|c|c|c|}
\hline Insect stage & $\mathbf{R}^{2}$ & $\mathbf{R}$ & P-value & Climatic variable \\
\hline \multicolumn{5}{|l|}{ Larvae } \\
\hline \multirow[t]{2}{*}{2009} & 0.352 & 0.593 & 0.490 & Control \\
\hline & 0.277 & 0.526 & 0.633 & Delayed \\
\hline \multirow[t]{2}{*}{2010} & 0.427 & 0.654 & 0.355 & Control \\
\hline & 0.375 & 0.613 & 0.446 & Delayed \\
\hline \multicolumn{5}{|l|}{ Pupae } \\
\hline \multirow[t]{2}{*}{2009} & 0.448 & 0.670 & 0.320 & Control \\
\hline & 0.266 & 0.516 & 0.653 & Delayed \\
\hline \multirow[t]{2}{*}{2010} & 0.250 & 0.500 & 0.685 & Control \\
\hline & 0.508 & 0.713 & 0.232 & Delayed \\
\hline \multicolumn{5}{|l|}{ Adult } \\
\hline \multirow[t]{2}{*}{2009} & 0.827 & 0.909 & $0.008^{* *}$ & Max. $\mathrm{T}^{\circ} \mathrm{C}, \mathrm{RH}$ \\
\hline & 0.837 & 0.915 & $0.007^{* *}$ & Delayed (RF) \\
\hline \multirow[t]{2}{*}{2010} & 0.707 & 0.841 & 0.048 & Control (RH) \\
\hline & 0.510 & 0.714 & 0.230 & Delayed \\
\hline \multicolumn{5}{|l|}{ Parasitoid } \\
\hline \multirow[t]{2}{*}{2009} & 0.909 & 0.954 & $0.001^{* *}$ & Max. $\mathrm{T}^{\circ} \mathrm{C}, \mathrm{RF}, \mathrm{RH}$ \\
\hline & 0.607 & 0.779 & 0.119 & Delayed \\
\hline \multirow[t]{2}{*}{2010} & 0.291 & 0.540 & 0.605 & Control \\
\hline & 0.708 & 0.814 & $0.047^{* *}$ & Min. $\mathrm{T}^{\circ} \mathrm{C}$ \\
\hline \multicolumn{5}{|l|}{ Predator } \\
\hline \multirow[t]{2}{*}{2009} & 0.604 & 0.777 & 0.122 & Control \\
\hline & 0.388 & 0.623 & 0.423 & Delayed \\
\hline \multirow[t]{2}{*}{2010} & 0.596 & 0.772 & 0.130 & Control \\
\hline & 0.817 & 0.904 & $0.001^{* *}$ & Min. $\mathrm{T}^{\circ} \mathrm{C}$ \\
\hline \multicolumn{5}{|c|}{ Grouped leaf miner } \\
\hline \multirow[t]{2}{*}{2009} & 0.725 & 0.851 & $0.039^{* *}$ & RF \\
\hline & 0.864 & 0.747 & $0.029^{* *}$ & RF \\
\hline \multirow[t]{2}{*}{2010} & 0.519 & 0.721 & 0.271 & Control \\
\hline & 0.599 & 0.774 & 0.127 & Delayed \\
\hline
\end{tabular}

${ }^{* *}$ Significance $\mathrm{P} \leq 0.05$. Control—climatic variable from month of collection; Delayed-climatic variable with a delayed month in relation to collection; $\mathrm{R}^{2}$ — coefficient of determination; $\mathrm{R}$-correlation coefficient. 
Table 2. Rainy season relationship between mean weather factors and leaf miner stages (larvae, pupae, adult), predators and parasitoids for 2009-2010.

\begin{tabular}{|c|c|c|c|c|}
\hline Insect stage & $\mathbf{R}^{2}$ & $\mathbf{R}$ & P-value & $\begin{array}{l}\text { Climatic } \\
\text { variable }\end{array}$ \\
\hline \multicolumn{5}{|l|}{ Larvae } \\
\hline \multirow[t]{2}{*}{2009} & 0.655 & 0.809 & 0.571 & Control \\
\hline & 0.099 & 0.314 & 0.990 & Delayed \\
\hline \multirow[t]{2}{*}{2010} & 0.097 & 0.311 & 0.991 & Control \\
\hline & 0.957 & 0.978 & 0.084 & Delayed \\
\hline \multicolumn{5}{|l|}{ Pupae } \\
\hline \multirow[t]{2}{*}{2009} & 0.625 & 0.791 & 0.609 & Control \\
\hline & 0.772 & 0.878 & 0.404 & Delayed \\
\hline \multirow[t]{2}{*}{2010} & 0.927 & 0.963 & 0.141 & Control \\
\hline & 0.732 & 0.855 & 0.465 & Delayed \\
\hline \multicolumn{5}{|l|}{ Adult } \\
\hline \multirow[t]{2}{*}{2009} & 0.911 & 0.954 & 0.170 & Control \\
\hline & 0.930 & 0.964 & 0.135 & Delayed \\
\hline \multirow[t]{2}{*}{2010} & 0.735 & 0.857 & 0.460 & Control \\
\hline & 0.900 & 0.949 & 0.190 & Delayed \\
\hline \multicolumn{5}{|l|}{ Parasitoid } \\
\hline \multirow{2}{*}{2009} & 0.519 & 0.720 & 0.731 & Control \\
\hline & 0.852 & 0.923 & 0.273 & Delayed \\
\hline \multirow[t]{2}{*}{2010} & 0.890 & 0.943 & 0.208 & Control \\
\hline & 0.633 & 0.796 & 0.599 & Delayed \\
\hline \multicolumn{5}{|l|}{ Predator } \\
\hline \multirow[t]{2}{*}{2009} & 0.986 & 0.993 & $0.029^{* *}$ & $\mathrm{RH}$ \\
\hline & 0.525 & 0.962 & 0.144 & Delayed \\
\hline \multirow[t]{2}{*}{2010} & 0.292 & 0.540 & 0.915 & Control \\
\hline & 0.745 & 0.863 & 0.445 & Delayed \\
\hline \multicolumn{5}{|c|}{ Grouped leaf miner } \\
\hline \multirow[t]{2}{*}{2009} & 0.615 & 0.785 & 0.621 & Control \\
\hline & 0.563 & 0.751 & 0.682 & Delayed \\
\hline \multirow[t]{2}{*}{2010} & 0.386 & 0.622 & 0.851 & Control \\
\hline & 0.575 & 0.758 & 0.670 & Delayed \\
\hline
\end{tabular}

${ }^{* *}$ Significance $\mathrm{P} \leq 0.05$.

Table 3. Dry season relationship between mean weather factors and leaf miner stages (larvae, pupae, adult), predators and parasitoids for 2009-2010.

\begin{tabular}{|c|c|c|c|c|}
\hline $\begin{array}{l}\text { Insect } \\
\text { stage }\end{array}$ & $\mathbf{R}^{2}$ & $\mathbf{R}$ & P-value & $\begin{array}{l}\text { Climatic } \\
\text { variable }\end{array}$ \\
\hline Larvae & $\begin{array}{l}1.0 \\
1.0\end{array}$ & $\begin{array}{l}1.0 \\
1.0\end{array}$ & $\begin{array}{l}0.000^{* *} \\
0.000^{* *}\end{array}$ & $\begin{array}{l}\text { Max. } \mathrm{T}^{\circ} \mathrm{C} \\
\operatorname{Max} . \mathrm{T}^{\circ} \mathrm{C}\end{array}$ \\
\hline Pupae & $\begin{array}{l}1.0 \\
1.0\end{array}$ & $\begin{array}{l}1.0 \\
1.0\end{array}$ & $\begin{array}{l}0.000^{* *} \\
0.000^{* *}\end{array}$ & $\begin{array}{l}\operatorname{Max} . \mathrm{T}^{\circ} \mathrm{C} \\
\operatorname{Max} . \mathrm{T}^{\circ} \mathrm{C}\end{array}$ \\
\hline Adult & $\begin{array}{l}1.0 \\
1.0\end{array}$ & $\begin{array}{l}1.0 \\
1.0\end{array}$ & $\begin{array}{l}0.000^{* *} \\
0.000^{* *}\end{array}$ & $\begin{array}{l}\operatorname{Max} . \mathrm{T}^{\circ} \mathrm{C} \\
\operatorname{Max} . \mathrm{T}^{\circ} \mathrm{C}\end{array}$ \\
\hline Parsitoid & $\begin{array}{l}1.0 \\
1.0\end{array}$ & $\begin{array}{l}1.0 \\
1.0\end{array}$ & $\begin{array}{l}0.000^{* * *} \\
0.000^{* *}\end{array}$ & $\begin{array}{l}\operatorname{Max} . \mathrm{T}^{\circ} \mathrm{C} \\
\operatorname{Max} . \mathrm{T}^{\circ} \mathrm{C}\end{array}$ \\
\hline Predator & $\begin{array}{l}1.0 \\
1.0\end{array}$ & $\begin{array}{l}1.0 \\
1.0\end{array}$ & $\begin{array}{l}0.000^{* *} \\
0.000^{* *}\end{array}$ & $\begin{array}{l}\operatorname{Max} . \mathrm{T}^{\circ} \mathrm{C} \\
\operatorname{Max} . \mathrm{T}^{\circ} \mathrm{C}\end{array}$ \\
\hline $\begin{array}{l}\text { Grouped } \\
\text { leaf miner }\end{array}$ & $\begin{array}{l}1.0 \\
1.0\end{array}$ & $\begin{array}{l}1.0 \\
1.0\end{array}$ & $\begin{array}{l}0.000^{* *} \\
0.000^{* *}\end{array}$ & $\begin{array}{l}\operatorname{Max} . \mathrm{T}^{\circ} \mathrm{C} \\
\operatorname{Max} . \mathrm{T}^{\circ} \mathrm{C}\end{array}$ \\
\hline
\end{tabular}

Significance $\mathrm{P} \leq 0.05$.

tablishment; and initiation of insect outbreaks [21]. Among the weather elements, temperature, humidity, and wind play the major roles in insect life [22]. The leaf miner adult is the only stage that lives outside the mines and its external environment, and it's the stage that is in
Table 4. Relationship between mean weather factors and leaf miner stages (larvae, pupae, adult) for 1976-1980.

\begin{tabular}{|c|c|c|c|c|}
\hline Insect stage & $\mathbf{R}^{2}$ & $\mathbf{R}$ & P-value & $\begin{array}{l}\text { Climatic } \\
\text { variable }\end{array}$ \\
\hline \multicolumn{5}{|l|}{ Larvae } \\
\hline \multirow[t]{2}{*}{1976} & 0.699 & 0.836 & 0.052 & $\operatorname{Max} . \mathrm{T}^{\circ} \mathrm{C}$ \\
\hline & 0.719 & 0.848 & $0.041^{* *}$ & $\operatorname{Max} . \mathrm{T}^{\circ} \mathrm{C}$ \\
\hline \multirow[t]{2}{*}{1977} & 0.477 & 0.692 & 0.273 & Control \\
\hline & 0.621 & 0.788 & 0.107 & Delayed \\
\hline \multirow[t]{2}{*}{1978} & 0.433 & 0.658 & 0.345 & Control \\
\hline & 0.494 & 0.703 & 0.252 & Delayed \\
\hline \multirow[t]{2}{*}{1979} & 0.523 & 0.723 & 0.213 & Control \\
\hline & 0.340 & 0.583 & 0.512 & Delayed \\
\hline \multirow[t]{2}{*}{1980} & 0.714 & 0.845 & $0.044^{* *}$ & Min. $\mathrm{T}^{\circ} \mathrm{C}$ \\
\hline & 0.465 & 0.682 & 0.294 & Delayed \\
\hline \multicolumn{5}{|l|}{ Pupae } \\
\hline \multirow[t]{2}{*}{1976} & 0.751 & 0.867 & $0.028^{* *}$ & Max. \& Min. $\mathrm{T}^{\circ} \mathrm{C}$ \\
\hline & 0.779 & 0.883 & $0.019^{* *}$ & $\operatorname{Max} . \mathrm{T}^{\circ} \mathrm{C}$ \\
\hline \multirow[t]{2}{*}{1977} & 0.371 & 0.609 & 0.454 & Control \\
\hline & 0.343 & 0.585 & 0.507 & Delayed \\
\hline \multirow[t]{2}{*}{1978} & 0.305 & 0.552 & 0.579 & Control \\
\hline & 0.344 & 0.586 & 0.504 & Delayed \\
\hline \multirow[t]{2}{*}{1979} & 0.223 & 0.473 & 0.736 & Control \\
\hline & 0.714 & 0.845 & $0.044^{* *}$ & RF, Max. $T^{\circ} \mathrm{C}$ \\
\hline \multirow[t]{2}{*}{1980} & 0.214 & 0.463 & 0.752 & Control \\
\hline & 0.793 & 0.890 & $0.015^{* *}$ & RF, Max. \& Min. $\mathrm{T}^{\circ} \mathrm{C}$ \\
\hline \multicolumn{5}{|l|}{ Adult } \\
\hline \multirow[t]{2}{*}{1976} & 0.330 & 0.575 & 0.530 & Control \\
\hline & 0.188 & 0.434 & 0.800 & Delayed \\
\hline \multirow[t]{2}{*}{1977} & 0.223 & 0.472 & 0.737 & Control \\
\hline & 0.041 & 0.204 & 0.987 & Delayed \\
\hline \multirow[t]{2}{*}{1978} & 0.257 & 0.507 & 0.672 & Control \\
\hline & 0.112 & 0.335 & 0.919 & Delayed \\
\hline \multirow[t]{2}{*}{1979} & 0.316 & 0.562 & 0.557 & Control \\
\hline & 0.242 & 0.492 & 0.701 & Delayed \\
\hline \multirow[t]{2}{*}{1980} & 0.246 & 0.496 & 0.693 & Control \\
\hline & 0.322 & 0.567 & 0.547 & Delayed \\
\hline \multicolumn{5}{|l|}{$\begin{array}{l}\text { Grouped } \\
\text { leaf miner }\end{array}$} \\
\hline \multirow[t]{2}{*}{1976} & 0.378 & 0.615 & 0.441 & Control \\
\hline & 0.062 & 0.248 & 0.973 & Delayed \\
\hline \multirow[t]{2}{*}{1977} & 0.335 & 0.579 & 0.520 & Control \\
\hline & 0.306 & 0.553 & 0.577 & Delayed \\
\hline \multirow[t]{2}{*}{1978} & 0.280 & 0.530 & 0.626 & Control \\
\hline & 0.072 & 0.269 & 0.964 & Delayed \\
\hline \multirow[t]{2}{*}{1979} & 0.300 & 0.548 & 0.588 & Control \\
\hline & 0.165 & 0.406 & 0.839 & Delayed \\
\hline \multirow[t]{2}{*}{1980} & 0.130 & 0.361 & 0.894 & Control \\
\hline & 0.535 & 0.731 & 0.197 & Delayed \\
\hline
\end{tabular}

${ }^{* *}$ Significance $\mathrm{P} \leq 0.05$.

direct contact with weather factors.

The rainy season generally had lower leaf miner and parasitoid abundance for both 2009 and 2010. The higher parasitoid population could probably explain low leaf miner population. Rainfall could also lead to disruption in breeding and mortality of the leaf miner and micromischoides sp.

The dry season generally had higher leaf miner and predator abundance and lower parasitoid populations for both 2009 and 2010. Higher mean temperatures prevail at 
Table 5. Rainy season relationship between mean weather factors and leaf miner stages (larvae, pupae, adult) for 1976-1980.

\begin{tabular}{|c|c|c|c|c|}
\hline $\begin{array}{l}\text { Insect } \\
\text { stage }\end{array}$ & $\mathbf{R}^{2}$ & $\mathbf{R}$ & P-value & $\begin{array}{l}\text { Climatic } \\
\text { variable }\end{array}$ \\
\hline \multicolumn{5}{|l|}{ Larvae } \\
\hline \multirow[t]{2}{*}{1976} & 0.978 & 0.989 & $0.043^{* *}$ & RF \\
\hline & 0.764 & 0.874 & 0.416 & Delayed \\
\hline \multirow[t]{2}{*}{1977} & 0.710 & 0.842 & 0.496 & Control \\
\hline & 0.711 & 0.843 & 0.495 & Delayed \\
\hline \multirow[t]{2}{*}{1978} & 0.683 & 0.826 & 0.534 & Control \\
\hline & 0.589 & 0.768 & 0.653 & Delayed \\
\hline \multirow[t]{2}{*}{1979} & 0.938 & 0.968 & 0.121 & Control \\
\hline & 0.969 & 0.985 & $0.046^{* *}$ & $\mathrm{RH}, \mathrm{Max} . \&$ Min. $\mathrm{T}^{\circ} \mathrm{C}$ \\
\hline \multirow[t]{2}{*}{1980} & 0.600 & 0.775 & 0.640 & Control \\
\hline & 0.809 & 0.900 & 0.345 & Delayed \\
\hline \multicolumn{5}{|l|}{ Pupae } \\
\hline \multirow[t]{2}{*}{1976} & 0.857 & 0.926 & 0.265 & Control \\
\hline & 0.928 & 0.758 & 0.140 & Delayed \\
\hline \multirow[t]{2}{*}{1977} & 0.769 & 0.877 & 0.409 & Control \\
\hline & 0.574 & 0.758 & 0.670 & Delayed \\
\hline \multirow[t]{2}{*}{1978} & 0.857 & 0.926 & 0.265 & Control \\
\hline & 0.751 & 0.866 & 0.437 & Delayed \\
\hline \multirow[t]{2}{*}{1979} & 0.643 & 0.802 & 0.587 & Control \\
\hline & 0.988 & 0.994 & $0.024^{* *}$ & RF, RH, Max. $T^{\circ} \mathrm{C}$ \\
\hline \multirow[t]{2}{*}{1980} & 0.630 & 0.794 & 0.603 & Control \\
\hline & 0.979 & 0.989 & $0.042^{* *}$ & Max. $\mathrm{T}^{\circ} \mathrm{C}$ \\
\hline \multicolumn{5}{|l|}{ Adult } \\
\hline \multirow[t]{2}{*}{1976} & 0.799 & 0.894 & 0.361 & Control \\
\hline & 0.602 & 0.776 & 0.638 & Delayed \\
\hline \multirow[t]{2}{*}{1977} & 0.598 & 0.773 & 0.643 & Control \\
\hline & 0.709 & 0.842 & 0.497 & Delayed \\
\hline \multirow[t]{2}{*}{1978} & 0.806 & 0.898 & 0.351 & Control \\
\hline & 0.562 & 0.866 & 0.684 & Delayed \\
\hline \multirow[t]{2}{*}{1979} & 0.610 & 0.781 & 0.628 & Control \\
\hline & 0.827 & 0.910 & 0.315 & Delayed \\
\hline \multirow[t]{2}{*}{1980} & 0.717 & 0.847 & 0.486 & Control \\
\hline & 0.314 & 0.560 & 0.902 & Delayed \\
\hline \multicolumn{5}{|c|}{$\begin{array}{l}\text { Grouped } \\
\text { leaf miner }\end{array}$} \\
\hline \multirow[t]{2}{*}{1976} & 0.834 & 0.943 & 0.305 & Control \\
\hline & 0.344 & 586 & 0.882 & Delayed \\
\hline \multirow[t]{2}{*}{1977} & 0.292 & 0.540 & 0.915 & Control \\
\hline & 0.582 & 0.763 & 0.661 & Delayed \\
\hline \multirow[t]{2}{*}{1978} & 0.866 & 0.931 & 0.250 & Control \\
\hline & 0.526 & 0.725 & 0.724 & Delayed \\
\hline \multirow[t]{2}{*}{1979} & 0.652 & 0.808 & 0.574 & Control \\
\hline & 0.739 & 0.859 & 0.455 & Delayed \\
\hline \multirow[t]{2}{*}{1980} & 0.647 & 0.804 & 0.582 & Control \\
\hline & 0.769 & 0.877 & 0.408 & Delayed \\
\hline
\end{tabular}

Significance $\mathrm{P} \leq 0.05$.

this time and is the most important factor affecting leaf miner abundance (Table 3). Similar findings were made from records between 1976 and 1980 (Table 6). Al though many studies have concluded that insect pests will become more abundant as temperatures increase [23-25], there are many uncertainties involved, particularly in predicting the result of interacting effects of ris ing $\mathrm{CO}_{2}$ (and other greenhouse gases) and temperature. Significant correlations were found between leaf miner,
Table 6. Dry season relationship between mean weather factors and leaf miner stages (larvae, pupae, adult) for 1976-1980.

\begin{tabular}{|c|c|c|c|c|}
\hline $\begin{array}{l}\text { Insect } \\
\text { stage }\end{array}$ & $\mathbf{R}^{2}$ & $\mathbf{R}$ & P-value & $\begin{array}{l}\text { Climatic } \\
\text { variable }\end{array}$ \\
\hline \multirow{2}{*}{ Larvae } & 1.0 & 1.0 & $0.000^{* *}$ & $\operatorname{Max} . \mathrm{T}^{\circ} \mathrm{C}$ \\
\hline & 1.0 & 1.0 & $0.000^{* *}$ & $\operatorname{Max} . \mathrm{T}^{\circ} \mathrm{C}$ \\
\hline \multirow{2}{*}{ Pupae } & 1.0 & 1.0 & $0.000^{* *}$ & $\operatorname{Max} . \mathrm{T}^{\circ} \mathrm{C}$ \\
\hline & 1.0 & 1.0 & $0.000^{* *}$ & $\operatorname{Max} . \mathrm{T}^{\circ} \mathrm{C}$ \\
\hline \multirow{2}{*}{ Adult } & 1.0 & 1.0 & $0.000^{* *}$ & $\operatorname{Max} . \mathrm{T}^{\circ} \mathrm{C}$ \\
\hline & 1.0 & 1.0 & $0.000^{* *}$ & $\operatorname{Max} . \mathrm{T}^{\circ} \mathrm{C}$ \\
\hline Grouped & 1.0 & 1.0 & $0.000^{* *}$ & Max. $\mathrm{T}^{\circ} \mathrm{C}$ \\
\hline leaf miner & 1.0 & 1.0 & $0.000^{* *}$ & $\operatorname{Max} . \mathrm{T}^{\circ} \mathrm{C}$ \\
\hline
\end{tabular}

its natural enemies and both control and delayed weather variables. This indicates that the weather variables at both the month of collection and with a delayed month in relation to collection is critical for pest-weather evaluation.

\section{Conclusions}

It can be concluded that temperature, rainfall and relative humidity had an effect on the population of $C$. elaeidis, and this effect is manifested primarily in seasonal fluctuations in oil palm agroecosystems. Weather influenced the seasonal population dynamics of $C$. elaeidis, facilitating early season build-up on the oil palm host crop. Dry season months with resultant higher temperatures recorded higher population of $C$. elaeidis.

This study provides a better understanding of how insect pest population dynamics and seasonal weather variation mechanism can fine-tune pest management strategies and respond to pest attacks. This study has focused on ecological principles that are cheap and sustainable, relying on need/timing for control of insect pests of the oil palm. The importance of indigenous knowledge of the physical and biological environments of insect pests is emphasized and the need for collection of minimum data sets over several seasons in order to quantify key factors necessary for designing pest management programmes.

\section{Acknowledgements}

The project was partly funded by the Agricultural Research Council of Nigeria, Competitive Agricultural Research Grant Scheme (CARGS) RFA 4 No. 6. We thank Mr. M. Emmanuel for assisting with pest surveys throughout the study period and Mr. G. Mbaeyi for statistical analysis.

\section{REFERENCES}

[1] H. Wolda, “Insect Seasonality: Why?” Annual Review of 
Ecology and Systematics, Vol. 19, 1988, pp. 1-18.

[2] M. H. O. Pinheiro, R. Monteiro and O. Cesar, "Levantamento Fitossociológico da Floresta Estacional Semidecidual do Jardim Botânico Municipal de Bauru, São Paulo,” Naturalia, Vol. 27, 2002, pp. 145-164.

[3] Nigerian Environmental Study/Action Team (NEST), "Executive Summary of Five Sector Surveys on Nigeria's Vulnerability and Adaptation to Climate Change,” 2004, pp. 1-17.

[4] V. A. Drake, "The Influence of Weather and Climate on Agriculturally Important Insects: An Australia Perspective,” Australian Journal of Agricultural Research, Vol. 45, No. 3, 1994, pp. 487-509. http://dx.doi.org/10.1071/AR9940487

[5] R. D. Alward, J. K. Ketling and D. G. Milchunas, "Grassland Vegetation Changes and Noctunal Global Warming," Science, Vol. 283, No. 5399, 1999, pp. 229-231. http://dx.doi.org/10.1126/science.283.5399.229

[6] S. Godefroid and M. Tanghe, "Influence of Small Climatic Variations on the Species Composition of Roadside Grasslands,” Phytocoenologia, Vol. 30, No. 3-4, 2000, pp. 655-664.

[7] G. R. Walther, "Climatic Forcing on the Dispersal of Toxic Species,” Phytocoenologia, Vol. 30, No. 3-4, 2000, pp. 409-430.

[8] T. J. C. Beebee, “Amphibian Breeding and Climate,” Nature, Vol. 374, 1995, pp. 219-220. http://dx.doi.org/10.1038/374219a0

[9] T. H. Sparks and T. J. Yates, "The Effect of Spring Temperature on the Appearance Dates of British Butterflies 1883-1993,” Ecography, Vol. 20, 1997, pp. 368-374.

[10] J. L. Brown, S. H. Li and N. Bhagabati, "Long-Term Trend toward Earlier Breeding in an American Bird: A Response to Global Warming?" Proceedings of the National Academy Sciences of the USA, Vol. 96, No. 10, 1999, pp. 5565-5569. http://dx.doi.org/10.1073/pnas.96.10.5565

[11] C. D. Allen and D. D. Breshears, "Drought Induced Shift of a Forest Woodland Ecotone: Rapid Landscape Response to Climate Variation," Proceedings of the $\mathrm{Na}$ tional Academy Sciences of the USA, Vol. 95, No. 25, 1998, pp. 14839-14842. http://dx.doi.org/10.1073/pnas.95.25.14839

[12] O. Hoegh-Guldberg, "Sizing the Impact: Coral Reef Ecosystems as Early Casualties of Climate Change,” In: G. R. Walther, C. A. Burga and P. J. Edwards, Eds., Fingerprints of Climate Change-Adapted Behavior and Shifting Species Ranges, Kluver Academic Publication, New York and London, 2001, pp. 205-230. http://dx.doi.org/10.1007/978-1-4419-8692-4_13

[13] D. R. Easterling, J. L. Evans, P. Y. Groisman, T. R. Karl, K. E. Kunkel and P. Ambenje, "Observed Variability and Trends in Extreme Climate Events: A Brief Review,”
Bulletin of the American Meteorological Society, Vol. 81, No. 3, 2000, pp. 417-425. http://dx.doi.org/10.1175/1520-0477(2000)081<0417:OV ATIE>2.3.CO;2

[14] D. R. Easterling, G. A. Meehl, C. Parmesan, S. A. Changnon, T. R. Karl and L. Mearns, "Climate Extremes: Observations, Modeling and Impacts,” Science, Vol. 289, No. 5487, 2000, pp. 2068-2074. http://dx.doi.org/10.1126/science.289.5487.2068

[15] J. S. Bale, I. D. Hodkinson, W. Block, N. R. Webb, S. J. Coulson and A. T. Strathdee, "Life Strategies of Arctic Terrestrial Arthropods,” In: S. J. Woodin and M. Marguiss, Eds., The Ecology of Arctic Environments, Blackwell Science, Oxford, 1997, pp. 137-165.

[16] C. T. Ho and C. L. Teh, "Integrated Pest Management in Plantation Crops in Malaysia. Challenges and Realities (pp. 125-49),” In: E. Pushparajah, Ed., Proceedings of the 1997 International Planters Conference-Plantation Management for the $21^{\text {st }}$ Century (vol. 1), Incorporated Society of Planters, Kuala Lumpur, 1997, pp. 1-192.

[17] R. V. H. Corley and P. B. Tinker, “The Oil Palm,” Fourth Edition, Blackwell Science Ltd., 2003, 562 p.

[18] H. Vine, "Studies of Soil Profiles at the WAIFOR Main Station and Other Sites of Oil Palm Experiments," Journal of West African Institute Oil Palm Research, Vol. 1, No. 4, 1956, pp. 8-59.

[19] FAO-UNESCO, “Soil Map of the World,” Paris, 1990.

[20] A. G. Ojanuga, G. Lekwa and F. R. Akamigbo, "Survey Genesis and Classification of Acid Sands," Acid Sands of Southern Nigeria, Publication Monograph No. 1, 1981. pp. 1-18.

[21] D. E. Pedgley, "Concentration of Flying Insects by the Wind," Philosophical Transactions of the Royal Society of London, Series B, Biological Sciences, Vol. 328, 1990, pp. 113-135.

[22] H. S. Mavi and G. J. Tupper, "Agrometeorology: Principles and Applications of Climate Studies in Agriculture," 2005, 364 p.

[23] A. D. Watt, L. K. Ward and B. C. Eversham, "Invertebrates," In: M. G. R. Cannell and M. D. Hopper, Eds., The Greenhouse Effect and Terrestrial Ecosystems of the UK, Institute of Terrestrial Ecology Research Publication 4, HMSO, London, 1990, pp. 32-37.

[24] M. E. Cammell and J. D. Knight, "Effects of Climatic Change on the Population Dynamics of Crop Pests," Advances in Ecological Research, Vol. 22, 1992, pp. 117162. http://dx.doi.org/10.1016/S0065-2504(08)60135-X

[25] R. Harrington and I. P. Woiwod, "Insect Crop Pests and the Changing Climate,” Weather, Vol. 50, 1995, pp. 200208. http://dx.doi.org/10.1002/j.1477-8696.1995.tb06108.x 